\title{
Analytical Considerations in the Study of Spatial Patterns Arising from Nonlocal Interaction Effects ${ }^{\dagger}$
}

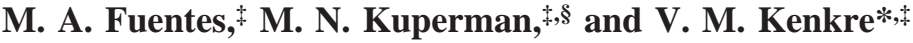 \\ Consortium of the Americas for Interdisciplinary Science and Department of Physics and Astronomy, \\ University of New Mexico, Albuquerque, New Mexico 87131, and Centro Atómico Bariloche and \\ Instituto Balseiro, 8400 S. C. de Bariloche, Argentina
}

Received: January 30, 2004; In Final Form: April 5, 2004

\begin{abstract}
Simple analytical considerations are applied to recently discovered patterns in a generalized Fisher equation. The generalization consists of the inclusion of nonlocal competition interactions among the constituents of the field exhibiting patterns. We show here how stability arguments yield a necessary condition for pattern formation involving the ratio of the pattern wavelength and the effective diffusion length of the individual constituents. We also remark on how a mode-mode coupling analysis may be developed that might be useful in shedding some light on the amplitude of the patterns.
\end{abstract}

\section{Introduction}

Patterns arise in many physical, chemical, and biological contexts. Chemical reactions, movement of granular assemblies, and populations in epidemics all exhibit patterns of various kinds. It has been shown recently that the Fisher equation used frequently for investigations of biological or ecological systems, when generalized to include spatially nonlocal competition interactions, leads to interesting patterns in the steady-state density. ${ }^{1,2}$ In this paper we attempt to shed some analytical light on the formation of these patterns. The considerations we discuss apply to chemical and physical systems as well as to ecological and biological entities. The original Fisher equation ${ }^{3,4}$ is

$$
\frac{\partial u(\vec{x}, t)}{\partial t}=D \nabla^{2} u(\vec{x}, t)+a u(\vec{x}, t)-b u^{2}(\vec{x}, t)
$$

where $u(\vec{x}, t)$ is the population density of the constituents under investigation (in a biological context, these might be bacteria) at position $\vec{x}$ and time $t$ and $D, a$, and $b$ are respectively the diffusion coefficient, population growth rate, and competition parameter. The generalized equation ${ }^{1,2}$ features competition interactions linking $u(\vec{x}, t)$ at point $\vec{x}$ with $u(\vec{y}, t)$ at point $\vec{y}$ through an influence function $f_{\sigma}(\vec{x}, \vec{y})$ of range $\sigma$

$$
\begin{aligned}
& \frac{\partial u(\vec{x}, t)}{\partial t}=D \nabla^{2} u(\vec{x}, t)+a u(\vec{x}, t)- \\
& b u(\vec{x}, t) \int_{\Omega} u(\vec{y}, t) f_{\sigma}(\vec{x}, \vec{y}) d y
\end{aligned}
$$

$\Omega$ being the domain of the system. The physical origin of the nonlocal aspect in the competition interaction is easy to understand. That interaction is mediated by the consumption of the same given resources by different individuals whose density $u(\vec{x}, t)$ measures. These resources can themselves be moving from place to place. One example is the diffusion of nutrients (and, indeed, in some cases the release of toxic substances) in the case wherein $u(\vec{x}, t)$ is the density of bacteria. The motion

\footnotetext{
† Part of the special issue "Gerald Small Festschrift".

$\doteqdot$ University of New Mexico.

$\S$ Centro Atómico Bariloche and Instituto Balseiro.
}

of the nutrients, if fast enough, can, in this manner, be a cause for the assumed nonlocality in the competition interaction.

The introduction of the finite-range competition interactions has been found ${ }^{1}$ to give rise to the emergence of patterns in the steady-state density $u(\vec{x})$ with the following features:

(i) No patterns appear in the two extremes of zero range, in which the generalization reverts to the Fisher equation, and full range, in which the population density is linked equally to all points in the domain.

(ii) The pattern structure depends crucially on features of the influence function, specifically its cutoff length and its width.

(iii) A critical quantity determining the formation of patterns appears to be the ratio of the cutoff length of the influence function to its width.

The first of these, that no patterns appear in the two extremes including, in particular, the full range case, has been proved analytically by one of the present authors earlier. ${ }^{2}$ An analytical investigation of the two other features is given below.

\section{Condition for Pattern Formation}

To address the question of why patterns form, we will follow a standard procedure ${ }^{5-7}$ to analyze the stability of a nonhomogeneous solution. We consider for simplicity a one-dimensional version of eq 2 and substitute in it

$$
u(x, t)=u_{0}+\epsilon \cos (k x) \exp (\varphi t)
$$

Here $u_{0}$ is the homogeneous steady-state solution $a / b$. Considering periodic boundary conditions and retaining only first-order terms in $\epsilon$, we obtain the following dispersion relation between the wavenumber $k$ of any mode of the pattern and the rate $\varphi$ at which it tends to grow:

$$
\left.\varphi=-D k^{2}-a \mathscr{R} k\right)
$$

In this expression, the influence function (assumed to be even) is represented by its cosine (Fourier) transform $\mathscr{R} k$ ) defined as

$$
\mathscr{A} k)=\int_{\Omega} \cos (k z) f_{\sigma}(z) \mathrm{d} z
$$


Stable steady-state patterns require that

$$
\lambda>2 \pi \sqrt{\frac{D}{-a \mathscr{R} \lambda)}}
$$

where $\lambda=2 \pi / k$ is the wavelength associated with the $k$ mode of the Fourier expansion of the pattern.

Condition (6) provides a necessary condition to check for the existence or absence of inhomogeneity, i.e., patterns, in the steady state. We see from (6) that the Fourier transform of the influence function at the wavelength under consideration should be negative for the patterns to appear and that its magnitude should be large enough. One way of understanding this condition is to recast it as requiring that $2 \pi$ times the "effective diffusion length" should be smaller than the wavelength for the patterns to occur. By the effective diffusion constant, we mean $D$ divided by $-\mathscr{A} \lambda)$, which is a factor decided by the influence function, and by the diffusion length, we mean the distance traversed diffusively in a time interval on the order of the inverse of the growth rate. If the influence function is smooth such as in the case of a Gaussian in an infinite domain, the Fourier transform is positive and no patterns appear. A cutoff in the influence function produces oscillations in the Fourier transform, which can go negative for certain wavelengths. The reported finding 1 that the cutoff nature appears necessary to pattern formation can be understood naturally in this way.

\section{Specific Examples}

Let us consider, in turn, three cases of the influence function that we have used in our earlier investigations: ${ }^{1}$ square, cutoff Gaussian, and intermediate.

First we take

$$
f(x-y)=\frac{1}{2 w}\{\theta[w-(x-y)] \theta[w+(x-y)]\}
$$

where $\theta$ is the Heaviside function. The influence function is thus a square of the cutoff range measured by $w$ from its center. We will consider the case here when the range $w$ is smaller than, or equal to, the domain length $L$. Equation 4 then involves an integral from 0 to $w$ and gives

$$
\varphi=-a \frac{\sin (k w)}{k w}-D k^{2}
$$

In terms of dimensionless parameters

$$
\begin{gathered}
\varphi^{\prime}=\varphi / a \\
k^{\prime}=k \sqrt{D / a} \\
\eta=w \sqrt{a / D}
\end{gathered}
$$

we have

$$
\varphi^{\prime}=-\frac{\sin \left(k^{\prime} \eta\right)}{k^{\prime} \eta}-k^{\prime}
$$

which we plot in Figure 1 for three different values (50, 10, and 2) of the ratio $\eta$ of the width to the diffusion length (not effective diffusion length). For the third case, there are no patterns: diffusion is strong enough to wash them out. For the intermediate case, patterns can occur with wavelengths corresponding to values of around $k^{\prime} \approx 0.4$, while for the $\eta=50$ case, they occur around $k^{\prime} \approx 0.1$.

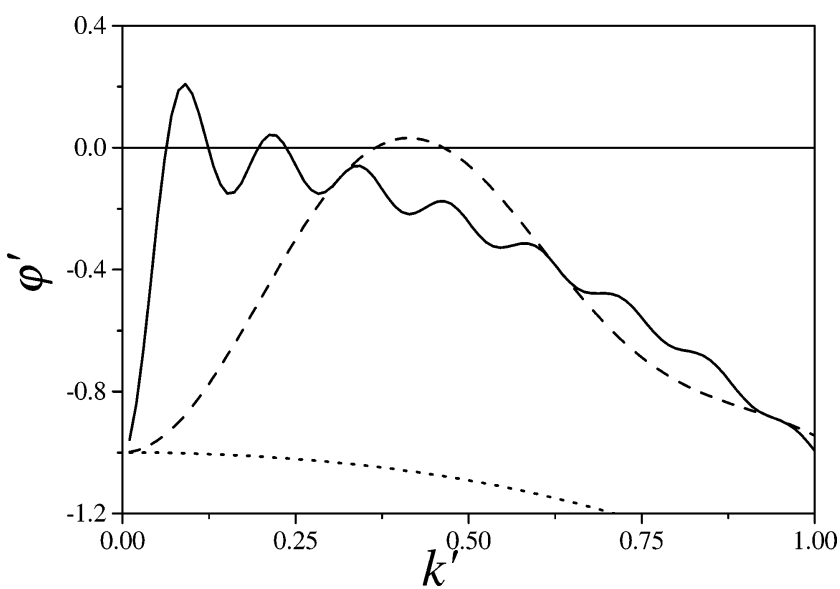

Figure 1. Dispersion relation (9) between the dimensionless growth exponent $\varphi^{\prime}$ and wavenumber $k^{\prime}$ plotted for different values of the ratio $\eta$ of the influence function range to the diffusion length (see the text). Values of $\eta$ are 50 (solid line), 10 (dashed line), and 2 (dotted line). Patterns appear for those values of $k^{\prime}$ for which $\varphi$ is positive.

The earlier finding ${ }^{1,2}$ that no patterns appear for extremes of the range of the influence function is clear from eq 9. As the influence width vanishes, i.e., as $\eta$ goes to zero, both terms in $\varphi$ are negative and there can be no steady-state patterns: we recover the solution for the local limit, corresponding to eq 1 , when $w \rightarrow 0$. Because the boundary conditions are periodic in a domain of length $L$, there are only the allowed values $k=$ $n \pi / L$ of the wavenumber. Therefore, in the opposite limit of full range, i.e., $w \rightarrow L$, the sine term vanishes, $\varphi^{\prime}=-k^{\prime}$, and again there are no patterns.

Precisely, the same qualitative behavior occurs for other nonsquare influence functions such as the Gaussian with a cutoff, i.e., for

$$
\begin{array}{r}
f(x-y)=\frac{1}{\sigma \sqrt{\pi} \operatorname{erf}(w / \sigma)} \exp \left[-\left(\frac{x-y}{\sigma}\right)^{2}\right]\{\theta[w-(x-y)] \\
\theta[w+(x-y)]\}
\end{array}
$$

We again consider the case when the cutoff length does not exceed the domain length. This leads to the Fourier transform of the influence function involving an integral from 0 to $w$. In these as well as other cases considered, it should be appreciated that the domain length $L$, if taken to be smaller than the cutoff length, becomes itself the cutoff length: factors such as $k w$ appearing in the Fourier transform become then $k L$ instead.

For this cutoff Gaussian case, the square case dispersion relation (8) is replaced by

$\varphi=-\frac{a \exp \left[-(k \sigma / 2)^{2}\right]}{2 \operatorname{erf}(w / \sigma)}\left[\operatorname{erf}\left(\frac{w}{\sigma}-\frac{i k \sigma}{2}\right)+\operatorname{erf}\left(\frac{w}{\sigma}+\frac{i k \sigma}{2}\right)\right]-D k^{2}$

The dimensionless version (9) is replaced by

$$
\varphi^{\prime}=-\frac{\exp \left(-k^{\prime} \beta^{2}\right)}{2 \operatorname{erf}(\alpha)}\left\{\operatorname{erf}\left(\alpha-i k^{\prime} \beta\right)+\operatorname{erf}\left(\alpha+i k^{\prime} \beta\right)\right\}-k^{\prime}
$$

Here $\alpha$ and $2 \beta$ are the ratios of the cutoff length to the range and of the range to the diffusion length respectively:

$$
\begin{gathered}
\alpha=w / \sigma \\
2 \beta=\sigma \sqrt{a / D}
\end{gathered}
$$

What is analogous to $\eta$ in the square case is their product $2 \alpha \beta$ 
$=w \sqrt{a / D}$. Plots that are essentially the same as those in Figure 1 can be drawn for this Gaussian case.

It is interesting to note that, while there is a single quantity $\eta$ in the square case, there are two quantities, $\alpha$ and $\beta$, in the cutoff Gaussian case. This arises from the fact that, although there are generally two lengths associated with any influence function, the cutoff length and the width, the latter is infinite for the square case. The width has been defined in ref 1 as being inversely proportional to the second derivative of the influence function evaluated at its central point and has been denoted by the symbol $\Sigma$. The cutoff length measures the distance beyond which the influence function is exactly zero and has been denoted $^{1}$ by $\xi_{\mathrm{c}}$. For the cutoff Gaussian, this $\xi_{\mathrm{c}}=x_{\mathrm{c}}=w$. The symbol $x_{\mathrm{c}}$ has been used in ref 1 and $w$ in the present paper. The width $\Sigma$ obeys $\Sigma=\sigma$ for the Gaussian case and $\Sigma=\infty$ for the square case.

The square and the cutoff Gaussian possess an abruptness feature that would not be present in a physical system. For this reason, we introduce a general function ${ }^{1,8}$ that is smooth and that we call the intermediate influence function:

$$
\begin{aligned}
& f(x-y)=\frac{\Gamma(1 / r+3 / 2)}{\sqrt{\pi} w \Gamma(1 / r+1)}\left[1-\frac{r(x-y)^{2}}{(2+3 r) \sigma^{2}}\right]^{1 / r} \theta[w- \\
& (x-y)] \theta[w+(x-y)]
\end{aligned}
$$

In addition to the smoothness property of this function, it has the feature that it reduces to a square or a Gaussian in the respective limits $r \rightarrow \infty$ and $r \rightarrow 0$. The cutoff length of the influence function is given by

$$
w=\sqrt{\frac{2+3 r}{r}} \sigma
$$

We will follow the notation

$$
v=1 / r+1 / 2
$$

and evaluate the Fourier transform of the influence function by calculating the integral ${ }^{9}$

$$
\begin{aligned}
& \frac{2 w^{-2 v} \Gamma(v+1)}{\sqrt{\pi} \Gamma(v+1 / 2)} \int_{0}^{w} \cos (k s)\left[w^{2}-s^{2}\right]^{v-1 / 2} \mathrm{~d} s= \\
&\left(\frac{2}{k w}\right)^{v} \Gamma(v+1) J_{v}(k w)
\end{aligned}
$$

for

$$
k>0, \quad w>0, \quad \operatorname{Re}[v]>1 / 2
$$

$\Gamma$ and $J$ being the gamma and Bessel functions, respectively. The dimensionless dispersion relation analogous to eq 9 is, for this general case,

$$
\varphi^{\prime}=-\left(\frac{2}{k^{\prime} \eta}\right)^{v} \Gamma(v+1) J_{v}\left(k^{\prime} \eta\right)-k^{2}
$$

Here, as in the square case, $\eta=w \sqrt{a / D}$ is the ratio of the influence function width to the diffusion length.

It is straightforward to obtain the two limits, square and Gaussian, from this dispersion result (15) for the intermediate influence function. In Figure 2, we plot the intermediate case for $v=1$ and see the same general behavior as that in the

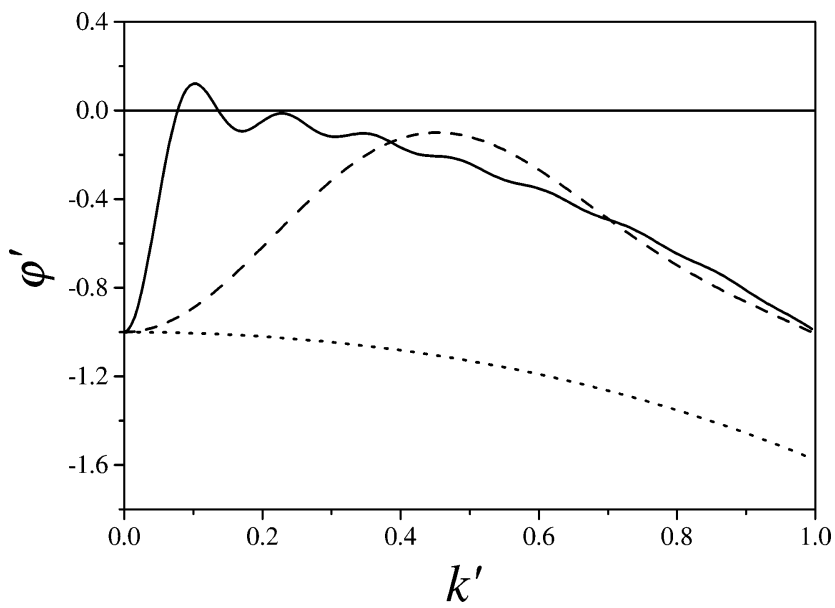

Figure 2. Dispersion relation (15) between the dimensionless growth exponent $\varphi^{\prime}$ and wavenumber $k^{\prime}$ plotted for the intermediate influence function. Values of $\eta$ are as in Figure 1: 50 (solid line), 10 (dashed line), and 2 (dotted line). Patterns appear for those values of $k^{\prime}$ for which $\varphi^{\prime}$ is positive.

Gaussian and square counterparts (see, e.g., Figure 1). Steadystate patterns appear only around $k^{\prime}=0.1$.

\section{Remarks}

We see that four lengths appear in this pattern formation problem, in addition to the (obvious) size of the domain: the pattern wavelength $\lambda$, the (cutoff) width $w$ and range $\sigma$ of the influence function, and the diffusion length, which is the distance covered by diffusing in a time proportional to the reciprocal of the growth rate $a$ and is proportional to $\sqrt{a / D}$. The precise manner in which the interplay of these four lengths gives rise to or suppresses the patterns is clear in the above discussion.

We note in passing that the Fourier mode expansion of $u(x, t)$

$$
u(\vec{x}, t)=\sum A_{n}(t) \cos \left(k_{n} x\right)
$$

in eq 2 , with $k_{n}=\pi n / L$, and the orthogonality properties of trigonometric functions yield separate equations for the $n=0$ mode

$$
\frac{\mathrm{d} A_{0}}{\mathrm{~d} t}=a A_{0}-b A_{0}{ }^{2}-b \sum_{n=1}^{\infty} \frac{A_{n}^{2}}{2} \mathscr{A}\left(k_{n}\right)
$$

and for other modes $n \neq 0$

$$
\begin{aligned}
\frac{\mathrm{d} A_{n}}{\mathrm{~d} t}= & -D k_{n}^{2} A_{n}+a A_{n}-b A_{0} A_{n}\left[1+\mathscr{A}\left(k_{n}\right)\right]- \\
& \left.b \sum_{j=1}^{n-1} \frac{A_{j} A_{n-j}}{2} \mathscr{A} k_{j}\right)-b \sum_{j=n+1}^{\infty} \frac{A_{j} A_{j-n}}{2}\left[\mathscr{A}\left(k_{j}\right)+\mathscr{A}\left(k_{j-n}\right)\right]
\end{aligned}
$$

Equations 17 and 18 are the complete set of equations for the evolution of the amplitudes of all modes in the nonlocal problem given by eq 2. A simultaneous solution of the algebraic equations obtained by putting the left-hand side of eqs 17 and 18 to zero leads to the evaluation of the amplitude of the involved modes. This would be a numerical undertaking. To make further progress analytically, we consider the situation near criticality (the onset of the pattern from a homogeneous background). We follow standard procedures ${ }^{5}$ to derive the 
expression for the evolution of the single mode, say $n=m$, for which $\varphi>0$ :

$$
\left.\frac{\mathrm{d} A_{m}}{\mathrm{~d} t}=-D k_{m}^{2} A_{m}+a A_{m}-b A_{0} A_{m}\left[1+\mathscr{A} k_{m}\right)\right]
$$

Taking into account that the evolution of $A_{0}$ near criticality will be

$$
\left.\frac{\mathrm{d} A_{0}}{\mathrm{~d} t}=a A_{0}-b A_{0}{ }^{2}-b \frac{A_{m}{ }^{2}}{2} \mathscr{A} k_{m}\right)
$$

we can get the stationary solution of eq 20 by putting its righthand side equal to zero, introduce the approximation of that solution correct to first order in $A_{m}$ into eq 19, and obtain for the mode forming the pattern

$$
\begin{aligned}
& \frac{\mathrm{d} A_{m}}{\mathrm{~d} t}=\left[-D k_{m}{ }^{2}-a \mathscr{A}\left(k_{m}\right)\right] A_{m}+\frac{b^{2} \mathscr{A}\left(k_{m}\right)}{2 a} A_{m}{ }^{3}= \\
& \varphi A_{m}+\frac{b^{2} \mathscr{A}\left(k_{m}\right)}{2 a} A_{m}{ }^{3}
\end{aligned}
$$

Equation 21 exhibits the expected exponential growth at the rate $\varphi$ along with the cubic saturation term, given that $\mathscr{A}\left(k_{\mathrm{m}}\right)<$ 0 at criticality.

We have also studied a number of additional features of this problem, including influence functions that do not peak at the center, and shown that the sharp cutoff requirement discussed in the present paper may be relaxed for such cases. Thus, while a single Gaussian influence function peaking at the origin will certainly not give rise to the patterns we have discussed, because the Fourier transform cannot become negative, a symmetric sum of two displaced Gaussians will give rise to patterns. ${ }^{10}$ We have also studied the time evolution of patterns for a variety of cases in two-dimensional as well as one-dimensional systems. These and related studies, including possible connections of our analysis to more recent work on patterns, ${ }^{11,12}$ will be reported in a future publication.

Acknowledgment. This work is supported in part by the Los Alamos National Laboratory via a grant made to the University of New Mexico (Consortium of the Americas for Interdisciplinary Science), by the NSF's Division of Materials Research via Grant DMR0097204, by the NSF's International Division via Grant INT-0336343, and by DARPA-N00014-031-0900.

\section{References and Notes}

(1) Fuentes, M. A.; Kuperman, M. N.; Kenkre, V. M. Phys. Rev. Lett. 2003, 91, 15 .

(2) Kenkre, V. M. In Patterns, Noise, and the Interplay of Nonlinearity and Complexity; Kenkre, V. M., Lindenberg, K., Eds.; Proceedings of the PASI on Modern Challenges in Statistical Mechanics; AIP: New York, 2003.

(3) Fisher, R. A. Ann. Eugen. London 1937, 7, 355-369.

(4) Murray, J. D. Mathematical Biology; Springer-Verlag: Berlin, 1993.

(5) Nicolis, G. Introduction to non linear science; Cambridge University Press: Cambridge, U.K., 1995.

(6) Rabinovich, M. I.; Ezersky, A. B.; Weidman, P. D. The Dynamics of Patterns; World Scientific: London, 2000.

(7) Newell, A. C. In Patterns Formation in the Physical and Biological Science; Nijhout, H. F., Nadel, L., Stein, D. L., Eds.; Lecture Notes of the Santa Fe Institute; Addison-Wesley: Reading, MA, 1997; Vol. V.

(8) Tsallis, C. J. Stat. Phys. 1988, 52, 449.

(9) Gradshteyn, I. S.; Ryzhuk, I. M. Table of Integrals, Series, and Products, 5th ed.; Academic Press: New York, p 465.

(10) Fuentes, M. A.; Kenkre, V. M. Preprint, University of New Mexico, Albuquerque, NM.

(11) Billingham, J. Nonlinearity 2003, 17, 313-345.

(12) Sayama, H.; de Aguiar, M. A. M.; Bar-Yam, Y.; Baranger, M. Phys. Rev. E 2002, 65, 051919. 